\title{
Multiplicity study of exoplanet host stars
}

\author{
Markus Mugrauer, Ralph Neuhäuser \\ Christian Ginski and Thomas Eisenbeiss \\ Astrophysical Institute and University-Observatory Jena, Germany \\ email: markus@astro.uni-jena.de
}

Abstract. We present recent results of our ongoing multiplicity study of exoplanet host stars.

\section{New low-mass stellar companions of exoplanet host stars}

In our imaging campaign, carried out with SofI/NTT and UFTI/UKIRT, we directly detected so far several new companions of exoplanet host stars. Among them HD 3651 B the first T dwarf companion of an exoplanet host star (1, 2), HD $27442 \mathrm{~B}$ a white dwarf which is the secondary of the most evolved exoplanet host star system presently known (3), as well as the binary companion of HD 65216, whose B component is a low mass star, while HD $65216 \mathrm{C}$ is either a massive brown dwarf or a very low-mass star (4). Recently, we identified two new low-mass stellar companions of the exoplanet host stars HD 125612 and HD 212301. The co-moving companion of HD 125612 is a wide M4 dwarf $\left(0.18 \mathrm{M}_{\odot}\right)$, located about $4750 \mathrm{AU}$ south-east of its primary. The co-moving companion of HD 212301 is a close M3 dwarf $\left(0.35 \mathrm{M}_{\odot}\right)$, which we found at about $230 \mathrm{AU}$ north-west of the exoplanet host star. The binaries HD 125612 AB and HD $212301 \mathrm{AB}$ are two new members in the continuously growing list of exoplanet host star systems of which more than 40 are presently known (5).

\section{Lucky-Imaging search for close companions of exoplanet host stars}

We started a search for close stellar companions of exoplanet host stars at the Calar Alto Observatory in Spain, using the Lucky-Imaging (L-I) technique. The observations are carried out with the $2.2 \mathrm{~m}$ telescope and its L-I camera AstraLux in the I-band. We take several thousand images with integration times down to $30 \mathrm{~ms}$, and choose a total integration time of about $30 \mathrm{~min}$ per target. After standard data-reduction, our L-I pipeline measures the Strehl-ratios of all images, and then selects only those images with the highest Strehl-ratios (selection rates from 1 to 10\%). Finally, all selected images are shifted and combined. With the achieved AstraLux detection limit, beyond 1 arcsec ( $\sim 40 \mathrm{AU}$ of projected separation at the average distance of our targets), we are sensitive to all stellar companions (with $\mathrm{M}>0.08 \mathrm{M}_{\odot}$ ) around our targets. Hence, close stellar companions that remain invisible in seeing limited observations are clearly detectable.

\section{References}

Mugrauer, M., Seifahrt, A., Neuhäuser, R., \& Mazeh, T. 2006, MNRAS 373, L31

Mugrauer, M., Seifahrt, A., Neuhäuser, R., Mazeh, T., \& Schmidt, T. 2007, IAUS 240, 638

Mugrauer, M., Neuhäuser, R., \& Mazeh, T. 2007, A\&A 469, 755

Mugrauer, M., Seifahrt, A., \& Neuhäuser, R. 2007, MNRAS 378, 1328

Mugrauer, M. \& Neuhäuser, R. 2009, A\&SA 494, 373 